\title{
El conocimiento del sentido común. Una herramienta básica en el análisis social
}

The knowledge of common sense. A basic tool in social analysis

Rómulo E. Navarrete-Noble ${ }^{\mathrm{a}}$

\begin{abstract}
:
The present text analyzes critically the theoretical proposal of Peter L. Berger and Thomas Luckmann on the Social Construction of Reality (1991), taking as reference the knowledge of common sense.
\end{abstract}

Keywords:

daily life, language and face-to-face situation typifying schemes, limited areas of meaning, temporal structure, spatial structure

\section{Resumen:}

El presente texto analiza críticamente la propuesta teórica de Peter L. Berger y Thomas Luckmann sobre la Construcción Social de la Realidad (1991), tomando como referencia el conocimiento del sentido común.

\section{Palabras Clave:}

vida cotidiana, lenguaje y situación cara a cara esquemas tipificadores, zonas limitadas de significados, estructura temporal, estructura espacial

\section{Introducción}

El objetivo de la presente reseña consiste en analizar críticamente la propuesta teórica de Peter L. Berger y Thomas Luckmann sobre la Construcción Social de la Realidad (1991), en el estudio de las situaciones que muestren cómo desde el conocimiento del sentido común el sujeto construye diversas soluciones a los problemas que enfrenta en la vida diaria.

\section{Los conceptos}

Para comenzar podemos razonar la propuesta de Berger \& Luckmann a partir de dos niveles de análisis que nos permitan acercarnos la conocimiento del sentido común. Este conocimiento es el conocimiento que comparto con otros en las rutinas normales y auto evidentes de la vida cotidiana (Berger y Luckmann, 1991, p. 41). Es decir, es el conocimiento que todos los días ponemos en práctica y que muchas veces pasa desapercibido.

En un primer nivel encontramos los conceptos primarios o fundamentales. Dentro de los conceptos fundamentales podemos ubicar las nociones de vida cotidiana, lenguaje y situación cara a cara. Estos conceptos se caracterizan porque se encuentran estrechamente ligados entre sí, además que, de ellos se desprenden concepciones secundarias.

En un segundo nivel encontramos percepciones secundarias o derivadas, los cuales, como su nombre lo indica, adquieren sentido (realidad, concreción) a partir de conceptos primarios. Conceptos como esquemas tipificadores, zonas limitadas de significados, estructura temporal, estructura espacial. Estas nociones no son accesibles en primera instancia, pues se encuentras tras el entramado de las relaciones sociales. Los sociólogos son los que les dan sentido a estas concepciones en el análisis de la vida cotidiana. Ambos niveles de análisis tienen la singular característica de que se derivan uno del otro, es decir, que se anteceden uno al otro y los cambios en una concepción afectan la otra.

Ahora bien para analizar los planteamientos de teóricos Peter L. Berger y Thomas Luckmann, examinaremos las representaciones de la vida cotidiana. Nuestros autores nos dicen que la vida cotidiana se presenta como una realidad interpretada por los hombres y que para ellos tiene el significado subjetivo de un mundo coherente. “(...) 
la vida cotidiana se organiza alrededor de mi cuerpo y el ahora de mi presente" (Berger \& Luckmann, 1991, 36). Entonces, la vida cotidiana como instrumento de análisis nos permitirá apreciar en forma cercana la construcción de una realidad por medio de significados que los sujetos fabrican a través de objetivaciones que le dan sentido a la sociedad a la que pertenecen.

Ligado al concepto de vida cotidiana encontramos la noción de lenguaje el cual se constituye como el elemento fundamental en el análisis del conocimiento del sentido común. Esto es porque se instituye como "el sistema de signos más importante en la sociedad humana (...) además que, nos proporciona las objetivaciones indispensables y dispone el orden dentro del cual adquiere sentido y la vida cotidiana tiene significado para mí" (Berger \& Luckman, 1991, 55). Por ello, es a través del análisis lingüístico que podemos estudiar cómo se expresa y se da forma al sentido de la vida cotidiana, lo mismo que podemos observar las percepciones que se crean en la sociedad.

Junto a los conceptos de vida cotidiana y lenguaje encontramos la noción de interacción cara a cara. Al respecto, nuestros autores plantean que "la experiencia más importante que tengo de los otros se produce en la interacción cara a cara que es el prototipo de la interacción social y de la que se derivan los demás casos (Berger \& Luckmann, 1991, 46). De ahí la relación con las otras nociones y la importancia como elemento de análisis.

Mientras que, los elementos del segundo nivel, no menos importantes en la propuesta teórica de nuestros autores, son los conceptos secundarios o derivados. Estas nociones adquieren relevancia para entender como se le da sentido al conocimiento del sentido común. Según los autores nosotros aprendemos al otro por medio de esquemas tipificadores en las interacciones sociales que desarrollamos "La realidad de la vida cotidiana contiene esquemas tipificadores en cuyos términos los otros son aprendidos y tratados en encuentros cara a cara. De este modo puedo aprender al otro como hombre, como europeo, como cliente, como hombre jovial" (Berger \& Luckmann, 1991, 46). De esta forma, aprendo la realidad social en un continumm de tipificaciones que nos permiten hacer objetivaciones que le dan sentido a nuestras vidas.

Otros conceptos que ubicamos en el segundo nivel análisis y que resultan indispensables para entender la propuesta de nuestros autores son las nociones de estructura espacial y estructura temporal. La estructura espacial es periférica con respecto a nuestras consideraciones presentes. Mientras que la estructura temporal se caracteriza por ser una propiedad intrínseca de la conciencia del individuo a la que todos tenemos acceso. Así pues la sociedad a la que pertenezco le impone a nuestro tiempo interior ciertas secuencias de hechos con lo que logra marcar las pautas de nuestro tiempo. "El mundo de la vida cotidiana tiene su propia hora oficial que se da intersubjetivamente. Esta hora oficial puede entenderse como la interacción del tiempo cósmico con su calendario establecido socialmente según las secuencias temporales de la naturaleza" (Berger \& Luckmann, 1991, 44).

Derivado de las concepciones de estructura temporal y espacial encontramos la noción de Zonas limitadas de significado, las cuales se caracterizan por desviar la atención de la realidad de la vida cotidiana y se produce un cambio radical en la tensión de la conciencia. Ejemplo de esta zona limitadas de significado son la religión, pues muchas veces recúrremos a ella buscando la solución a nuestros problemas. Inconscientemente pensamos que la religión puede resolver mágicamente nuestras complicaciones sin detenernos a pensar en una solución práctica desde el sentido común.

\section{Conclusiones}

Es innegable que los conceptos propuestos en la teoría de la construcción social de la realidad de Berger y Luckmann se tornan como herramientas indispensables en el análisis del conocimiento del sentido común. Debido a que las nociones que nos proponen (vida cotidiana, lenguaje $y$ situación cara a cara esquemas tipificadores, zonas limitadas de significados, estructura temporal, estructura espacial) nos cercan a la perspectiva de los sujetos sociales. Asimismo, nos descubren una serie de situaciones de la vida social que no son perceptibles a simpe vista y que solo un estudio de la conducta social puede observar. Sin embargo, consideramos que aunque los conceptos plateados en esta teoría resultan elementales. No son suficientes para explicar la realidad social. En virtud de que "la realidad" es sumamente compleja, por lo que, una sola teoría resulta insuficiente para tratar de explicarla.

\section{Referencias}

[1]Berger, P \& Luckmann, (1991) La Construcción Social de la Realidad. Amorrortu Editores. Buenos Aires, Argentina. 